\title{
Chain-shaped plasmonic crystal for highly sensitive label-free biosensor
}

\author{
Hirotaka Yamada ${ }^{1}$, Daiki Kawasaki ${ }^{1}$, Shoma Aki ${ }^{1}$, Kenichi Maeno ${ }^{1}$, \\ Kenji Sueyoshi', Hideaki Hisamoto ${ }^{1}$ Tatsuro Endo ${ }^{1,2}$ \\ 1 Osaka Prefecture University, ${ }^{2}$ JST PREST \\ endo@chem.osakafu-u.ac.jp
}

\begin{abstract}
In this study, we designed and fabricated plasmonic crystal (PC) which has chain-shaped periodic metal nanostructures for excitation of the localized surface plasmon resonance (LSPR) at two different wavelength by changing polarization of incident light. PC absorbs light and generates enhanced electric field at specific wavelength depending on the size, shape, thickness, periodicity, and material. Here, we designed the PC consisted of intersectional short and long chain structures for applications using both visible and infrared lights. With this PC, the short chain structure can be used for label-free biosensing based on the detection of surrounding refractive index change by visible light. On the other hand, long chain structure is applicable in molecular collection by gradient force and thermal convection occurred by irradiation of infrared light. Based on these effects of chain shapes, PC structure proposed here is expected to be useful in label-free biosensors for low-concentration analytes in sample solution.
\end{abstract}

Key words: Biosensor, Plasmonics, Plasmonic Crystal, Electron beam lithography

\section{Introduction}

Plasmonic crystal (PC), which consists of periodic metal nanostructures, has specific optical characteristics derived from localized surface plasmon resonance (LSPR). The LSPR is resonance between collective oscillation of free electron and electromagnetic wave at specific wavelength. Based on this phenomenon, PC exhibits the absorption of light and generation of enhanced field at specific wavelength. Since the LSPR wavelength changes along with refractive index change caused by binding event such as an antigen-antibody reaction around metal nanostructures, PC have been studied for the development of label-free biosensor [1].

The optical characteristics of PC-based sensor such as LSPR peak wavelength and intensity of electric field, which are related with the sensitivity of PC, can be tuned by controlling the shape, size, and material of metal nanostructures. However, PC structure for high sensitivity detection using visible light is scarcely reported [2]. Furthermore, to detect low concentration of target molecules, the binding events have to occur at the specific region of $\mathrm{PC}$ with strongly excited electric field derived from LSPR. Hence, for developing the more sensitive biosensor using $\mathrm{PC}$, target molecules need to be trapped around the specific region on the PC. To overcome this problem, plasmonic optical tweezers applying gradient force of electric field and thermal convection have been studied for effective trapping of target molecules to the specific area of PC. Recently, plasmonic optical tweezers for collection of nanospheres and molecules have been reported [3]. However, trapping effect works at infrared wavelength, so it was difficult to detect molecules at visible wavelength while trapping molecules.

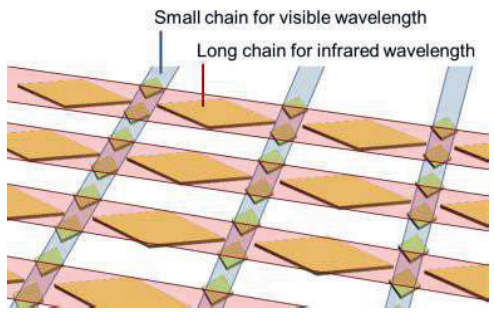

Fig. 1 Design of chain-shaped PC

Here, we report the design of chain-shaped plasmonic crystal (PC) (Fig. 1) for highly sensitive biosensor working at visible 
wavelength and plasmonic optical tweezers for trapping molecules working at infrared wavelength. This PC structure consists of short chain structure that resonates with light at visible wavelength and long chain structure that resonates with light at infrared wavelength. By changing polarization of incident light, two resonance wavelength can be switched. Based on this design, detection and trapping can be performed on the same PC.

In this study, we designed the Au-based PC which resonate at approximately $650 \mathrm{~nm}$ and $1300 \mathrm{~nm}$ using finite-difference time-domain (FDTD) simulation by changing the shape, size and periodicity of PC. Furthermore, we fabricated chain-shaped PC using electronbeam lithography, and the optical characteristics using polarized incident light was evaluated. From this evaluation, it was suggested that the chain-shaped PC fabricated here responded at different wavelength by changing the polarity of incident light.

\section{Design and fabrication of chain PC}

Au-based chain-shaped PC was designed by FDTD simulation for application using visible and infrared lights. In this case, PC structure exhibiting resonance at 650 and $1300 \mathrm{~nm}$ for each polarized incident light was investigated. Then, based on this simulation, chain-shaped PC was fabricated using electron beam lithography (Fig. 2).

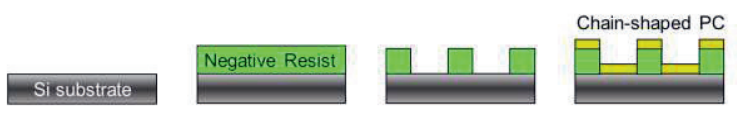

Spin-coating Electron beam lithography Au deposition

Fig.2 Fabrication procedure of chain-shaped PC

\section{Structural and optical characterization}

Structure (size and shape) of chain-shaped PC was observed using scanning electron microscopy (SEM). In addition, optical characteristics were evaluated using optical microspectroscopy. Here, differently-polarized white light was irradiated to the PC. Then, the reflection light was detected using spectrometer.

\section{Results and discussion}

Fig. 3 shows simulated absorption spectra and field intensity distribution of chain-shaped PC. Drastic changes of absorption wavelength and field intensity distribution upon changing the polarization of incident light were confirmed.

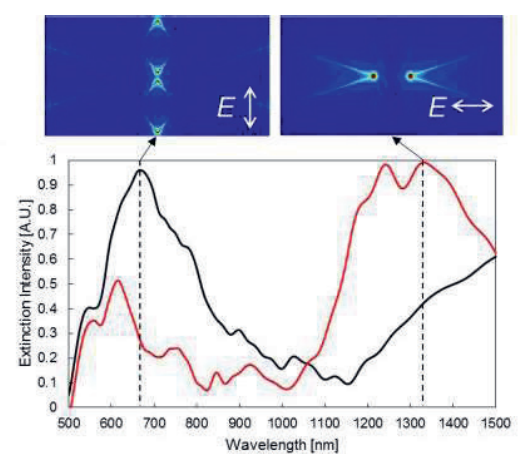

Fig.3 Simulated absorption spectra and field intensity distribution of chain-shaped PC

Fig. 4 and 5 show SEM image and absorption spectra at visible wavelength obtained for differently polarized incident light. As seen in Fig. 4, small and large chains were successfully fabricated by electron beam lithography and $\mathrm{Au}$ deposition.

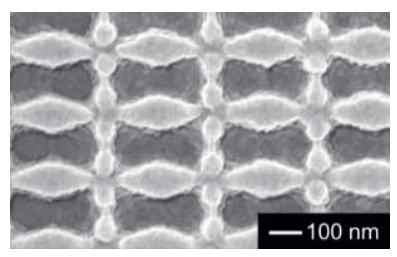

Fig.4 SEM image of fabricated chain-shaped PC

Furthermore, from Fig. 5, absorption peak change was seen by changing the polarization of incident light. These peaks at $716 \mathrm{~nm}$ and $682 \mathrm{~nm}$ corresponded to the LSPR occurring along the long axis and the short axis of short chain structure, respectively.

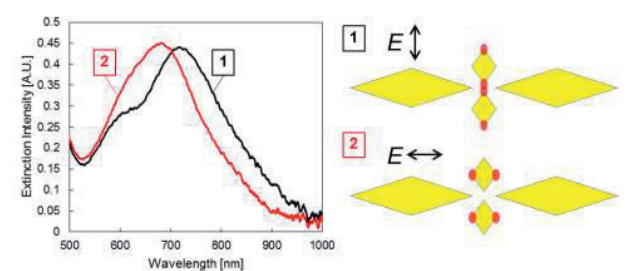

Fig. 5 Absorption spectra of fabricated chainshaped PC for differently polarized incident light

Based on these results, chain-shaped PC fabricated here can be applicable to highly sensitive label-free sensor using visible light. Investigation on the use of infrared light is currently underway.

\section{References}

[1] K. Nishiguchi, K. Sueyoshi, H. Hisamoto, and T. Endo, Jpn. J. Appl. Phys., 55, 08 RE02 (2016)

[2] H. Chen, X. Kou, Z. Yang, W. Ni, and J. Wang, Langmuir, 24, 5233-5237 (2008)

[3] T. Shoji and Y. Tsuboi, J. Phys. Chem. Lett., 5, 2957-2967 (2014) 